Return to the Manage Active Submissions page at http://spie.org/submissions/tasks.aspx and approve or disapprove this submission. Your manuscript will not be published without this approval. Please contact author_help@spie.org with any questions or concerns.

\title{
Laboratory investigation of an intensiometric dual FBG-based hybrid voltage sensor
}

\author{
Grzegorz Fusiek and Paweł Niewczas \\ Institute for Energy and Environment, Department of Electronic and Electrical Engineering, \\ University of Strathclyde, Glasgow G1 1XW, United Kingdom \\ Tel: +44 (0)141548 2609, Fax: +44 (0)141548 4872 \\ E-mail: g.fusiek@strath.ac.uk
}

\begin{abstract}
In this paper, we investigate experimentally the performance of a prototype optical voltage sensor utilizing a piezoelectric component and a dual FBG sensor combined with an intesiometric detection scheme. The sensor construction allows for the sensor interrogation using a single photodetector, and by eliminating spectral scanning devices, the measurement system benefits from a significant cost reduction and potentially improved speed of operation. Preliminary voltage characterization reveals that the proposed sensor is capable of reliable ac voltage measurement.
\end{abstract}

Key words: Optical voltage sensor, fiber Bragg gratings, piezoelectric transducer, voltage monitoring.

\section{INTRODUCTION}

The development of smart grid technology has been pursued by the electrical power industry in recent years to enable increased visibility, feedback, and control of grid operations and to improve its efficiency, reliability and cost-effectiveness. The requirements of voltage distortion monitoring, and voltage and power quality monitoring, contrasted with the difficulty of voltage harmonic transfer through instrument voltage transformers have been identified as monitoring problems in medium voltage (MV) and high voltage (HV) power networks [1], [2]. The inherent dielectric nature of fiber optic sensors combined with the use of optical fiber as an excellent communication medium make the fiber optic technology a desirable solution for smart grid applications. Although the optical voltage and current transducers have matured to the point that they meet the relevant industry standards, the optical sensor technology still represents a niche market. The main reason for this is the high cost of the existing optical measurement systems in comparison to the conventional equivalents.

In this paper, we determine experimentally the performance of a hybrid optical voltage sensor based on a combination of a piezoelectric transducer and an FBG sensor interrogated using a single photodetector. Strain/voltage measurements with a decoupled temperature-dependent wavelength shift capability and an intesiometric detection scheme allow to reduce the measurement system cost significantly. The sensor preliminary voltage characterizations is performed to assess the proposed sensor system capability to monitor ac voltage at high speed.

\section{VOLTAGE SENSOR DESIGN}

A piezoelectric material K-350 (Piezo Technologies) was utilized to fabricate a 6-layer stack. The thickness of each layer was $1.5 \mathrm{~mm}$, and the whole stack dimensions were $5 \times 10 \times 9 \mathrm{~mm}(\mathrm{H} \times \mathrm{W} \times \mathrm{L})$. The material has a longitudinal piezoelectric charge constant $d_{33}$ of $390 \mathrm{pm} / \mathrm{V}$ and a coefficient of thermal expansion (CTE) of $3.6 \mathrm{ppm} /{ }^{\circ} \mathrm{C}$ [3]. The first three layers were electrically connected in parallel and used as a voltage-to-strain converter while the remaining layers were shortcircuited and used as a temperature compensation component (Figure 1). In order to produce a dual-peak reflection spectrum a standard $10 \mathrm{~mm}$ FBG with a central wavelength at $1550.1 \mathrm{~nm}$ and a bandwidth of $0.25 \mathrm{~nm}$ was epoxied to the piezoelectric transducer in two stages using a two-component epoxy (EPOTEK 301). Firstly, half of the FBG was epoxied to the temperature compensating part of the stack and cured for two hours at $65{ }^{\circ} \mathrm{C}$ in a laboratory oven. After the components were cooled naturally down to room temperature, the other half of the FBG was then prestressed to split the single Bragg reflection, and thereafter epoxied to the other part of the piezoelectric stack. The curing process took place at room temperature for 24 hours. After this fabrication step, one of the end electrodes was attached to a rigid conductor 
Return to the Manage Active Submissions page at http://spie.org/submissions/tasks.aspx and approve or disapprove this submission. Your manuscript will not be published without this approval. Please contact author_help@spie.org with any questions or concerns.

supporting the sensor structure, while the second electrode was attached to a flexible conductor allowing for unrestricted expansion and contraction of the piezoelectric element.

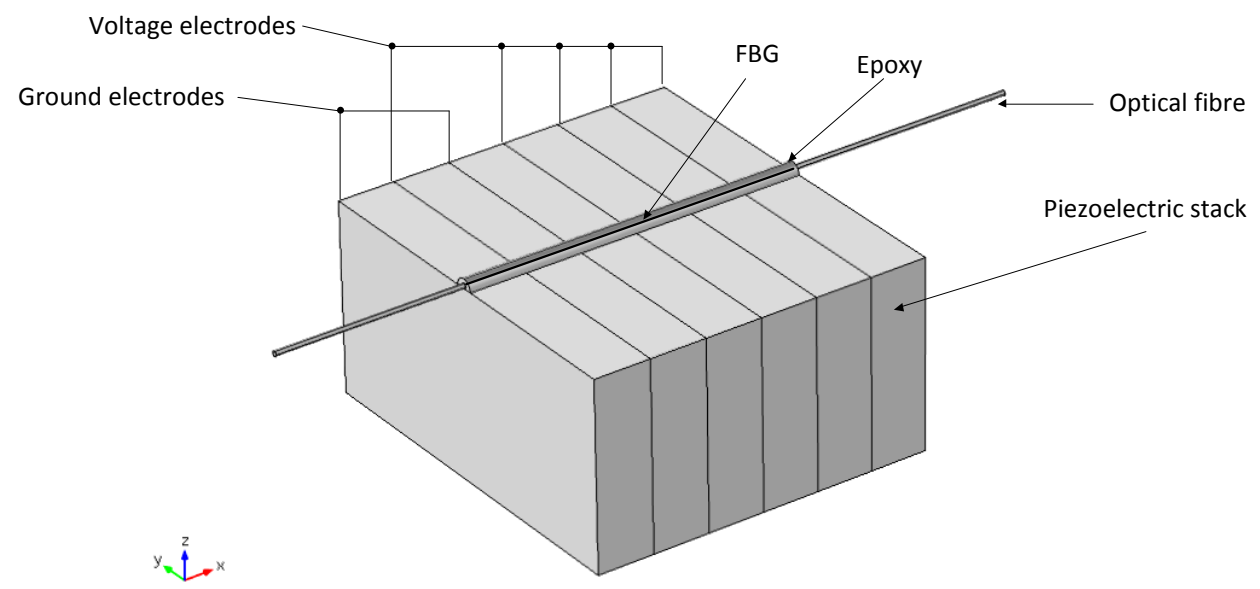

Figure 1. Conceptual voltage sensor construction.

In the absence of mechanical stress in the piezoelectric material, the strain (i.e. relative elongation $\Delta l / l$ ) induced by an external electric field is given by $\varepsilon=d_{33} \cdot E=d_{33} \cdot V / l$ where $d_{33}$ is the longitudinal piezoelectric charge constant, $E$ is the electric field, $V$ is the voltage applied across the piezoelectric element and $l$ is the length of the element. A relative change in the FBG peak wavelength, $\Delta \lambda_{B} / \lambda_{B}$, due to the change in strain, $\Delta \varepsilon$, and temperature, $\Delta T$, can be expressed by $\Delta \lambda_{B} / \lambda_{B}=$ $C_{\varepsilon} \cdot \Delta \varepsilon+C_{T} \cdot \Delta T$, where $C_{\varepsilon}$ and $C_{T}$ are the strain and temperature sensitivities and $\Delta \varepsilon=\left(\varepsilon+\varepsilon_{\text {thermal }}\right)$ is the sum of mechanical and thermal strain. Thermal strain produced in the material due to the temperature change, $\Delta T$, can be expressed by $\varepsilon_{\text {thermal }}=C T E \cdot \Delta T$, where $C T E$ is the coefficient of thermal expansion [4].

A dual-peak FBG can be considered as a combination of two overlapping single-peak FBGs, as shown in Figure 2. When such gratings are illuminated by a broadband light source, the optical power detected by a photodetector is equal to the integrated optical power over the combined reflected spectrum (the convolution curve in Figure 2). The spacing between FBGs, and hence, the degree to which they overlap, determines the total reflected optical power incident on the photodetector. Providing that half of the FBG is attached to the temperature compensating part (FBG1), and the other section of the FBG (FBG2) is attached to the other part of the piezoelectric element, the application of positive or negative voltage across the stack will result in the FBG2 peak shifting with respect to the FBG1 peak. This will be translated into the reflected optical power modulation detected by the photodetector. When a temperature change occurs, it causes both peaks to spectrally shift due to the thermal strain in the materials and thermo-optic effect in the fiber. The shift will be of the same magnitude and will not affect the reflected power distribution, allowing for temperature-independent strain/voltage measurements [4]. The depth of the power modulation will depend on the sensor sensitivity to voltage and the FBG bandwidth. Through careful design, the FBG shift can be tailored so that a specific modulation depth is reached at the full-scale voltage measurement (Figure 3) [4].

(a)

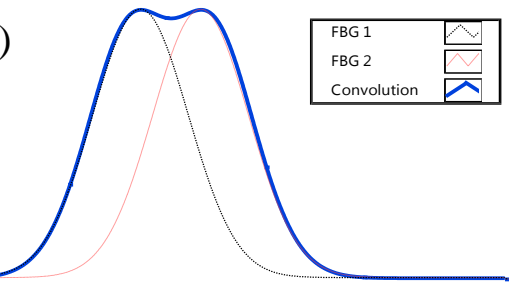

(b)

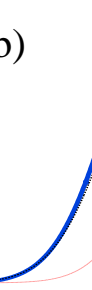

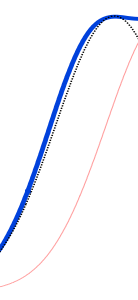

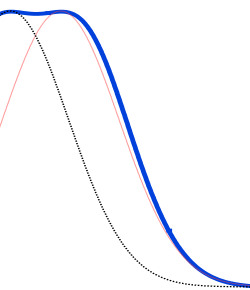

(c)

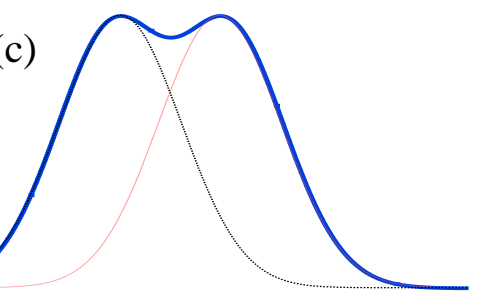

Figure 2. Optical power distribution between two FBGs: (a) nominal position of peaks (no voltage applied); (b) and (c) two extreme positions of a voltage modulated FBG peak. 
Return to the Manage Active Submissions page at http://spie.org/submissions/tasks.aspx and approve or disapprove this submission. Your manuscript will not be published without this approval. Please contact author_help@spie.org with any questions or concerns.

(a)

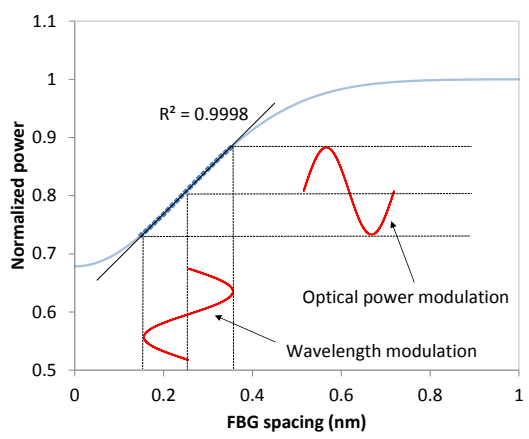

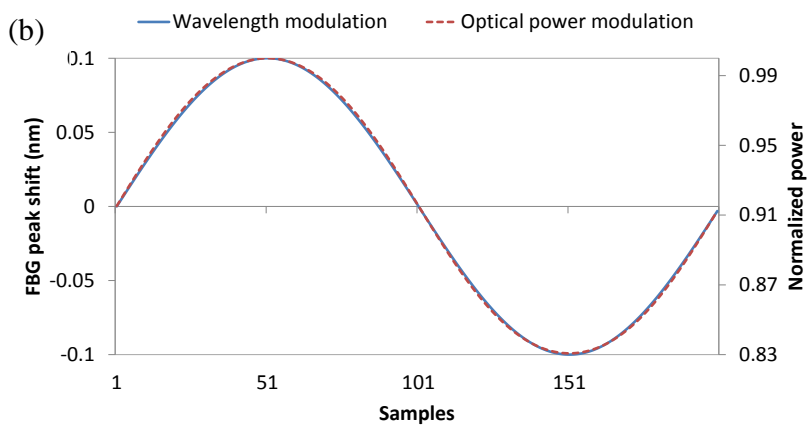

Figure 3. Normalized optical power levels versus FBG spacing for two gratings having equal reflections and bandwidths (a);

Optical power modulation in response to FBG peak wavelength modulation within the linearity region (b) [4].

\section{EXPERIMENTAL RESULTS}

To characterize the optical voltage sensor, the piezoelectric transducer was powered using a variac and an isolating voltage transformer (Figure 4), both capable of providing ac voltages up to $250 \mathrm{~V}_{\text {rms. }}$. During the experiments, the dual FBG sensor was illuminated by a broadband source (BBS) and the optical signals reflected from the sensor were converted to electrical signals by a single photodetector (PD). The output of the PD together with the reference voltage were captured by a 16-bit PXI data acquisition unit (National Instruments) and processed in a PC. To match the input impedance and voltage range of the PXI unit the reference voltage was measured through a specially designed $100 \mathrm{~V} / 1 \mathrm{~V}$ voltage divider followed by a buffer amplifier. The data acquisition rate was set to $100 \mathrm{kS} / \mathrm{s}$.

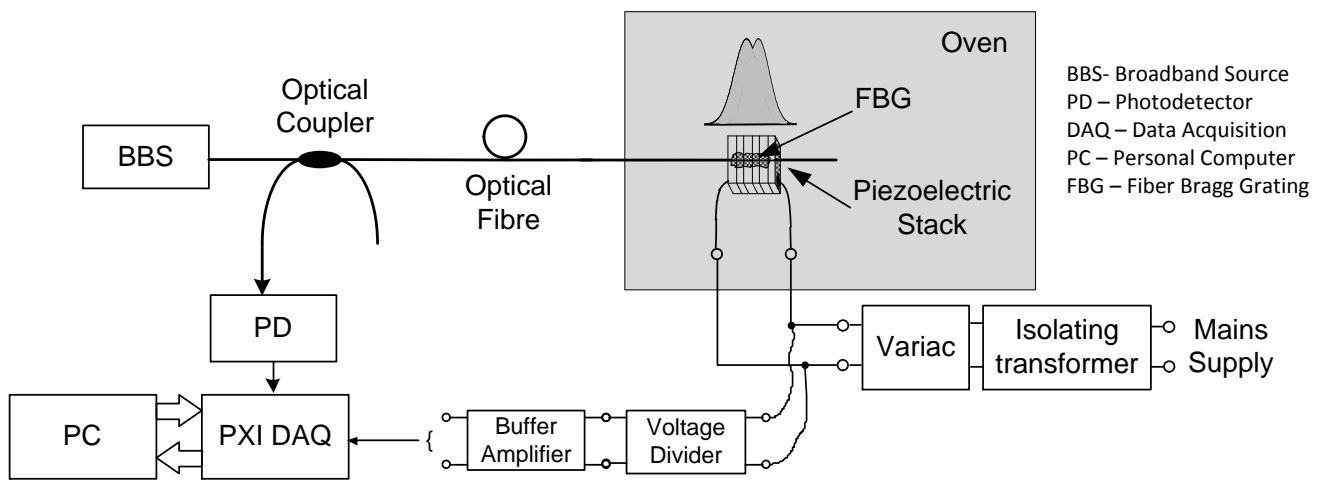

Figure 4. Experimental set-up for the voltage sensor testing procedure.

Spectra of the original and split FBG peaks are shown in Figure 5 (a), and a comparison between the sensor response when measuring an ac voltage of $200 \mathrm{~V}_{\mathrm{rms}}$ is shown in Figure 5 (b). A small phase shift between the signals can be noticed which stems from the hysteresis apparent in the piezoelectric material [5], [6]. Hysteretic behavior of the voltage sensor can be clearly seen in Figure 5 (c) where the ac PD output signals are plotted against the ac voltages applied to the stack at a temperature of $20^{\circ} \mathrm{C}$. The hysteresis widens and rotates slightly with the voltage magnitude increase. Similar behavior of the piezoelectric transducer was observed in the authors' previous work [5], [6]. The sensor rms characteristic is linear for the measured voltage range, as shown in Figure 5(d).

Similar experiments were performed at the temperature increased to $30{ }^{\circ} \mathrm{C}$. It can be seen from Figure 5 (d) that the sensitivity of the sensor decreased by nearly $50 \%$. Since the $d_{33}$ constant for the used piezoelectric material increases slightly with temperature [3], [5], this effect can be attributed to the failure of the epoxy cured at room temperature (Section 2). The epoxy under higher temperature softened releasing some of the initial prestress, and thus, amending the FBG peak separation causing a significant reduction in the optical power modulation at the same voltage. This issue will be addressed in the future work, for example by the application of different bonding agent. It should be noted, that in order to minimize voltage phase and amplitude measurement errors, a suitable hysteresis compensation methods could be implemented in the system [5], [6]. 
Return to the Manage Active Submissions page at http://spie.org/submissions/tasks.aspx and approve or disapprove this submission. Your manuscript will not be published without this approval. Please contact author_help@spie.org with any questions or concerns.
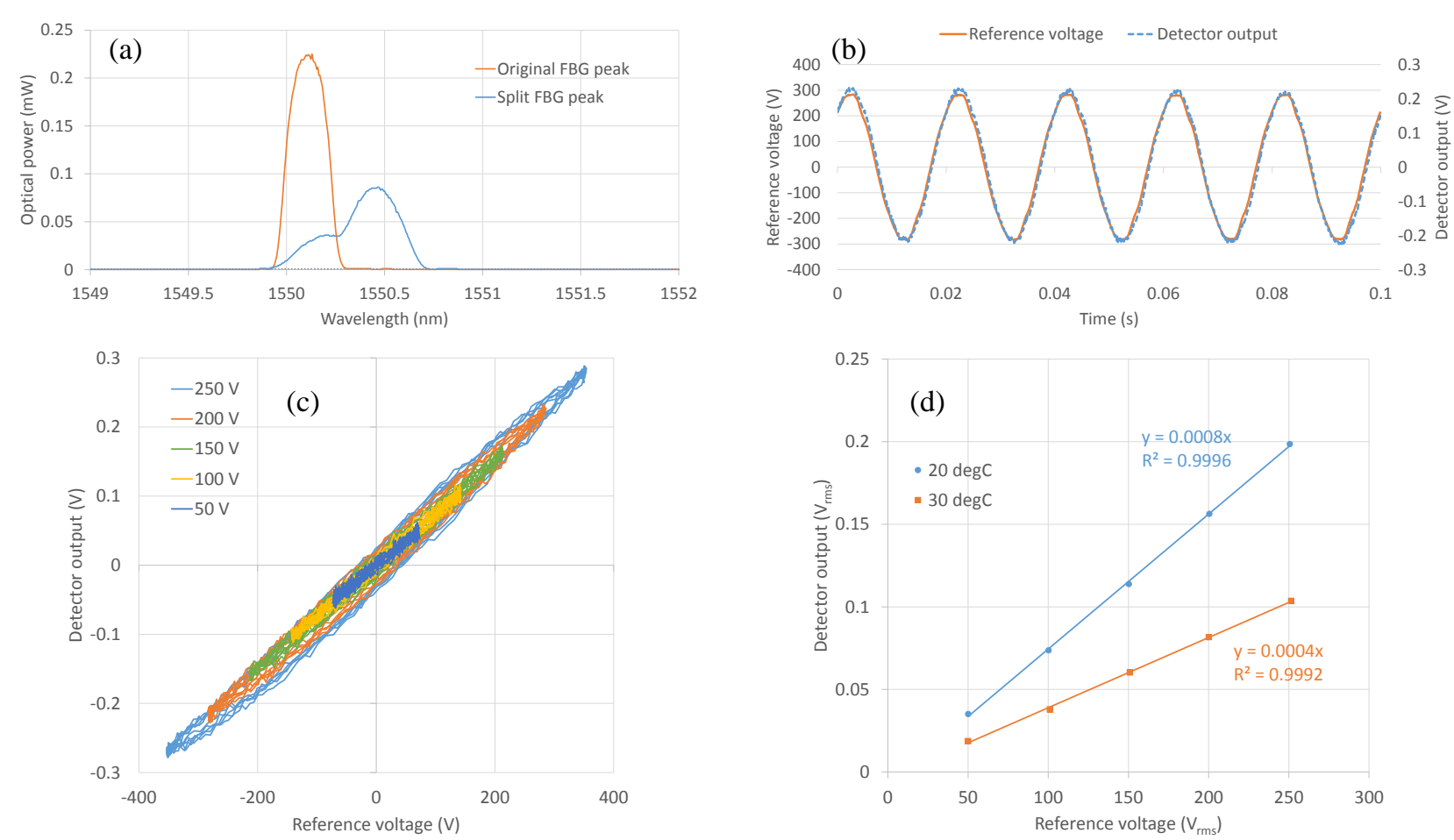

Figure 5. Voltage sensor characterization: (a) spectrum of the split FBG peak; (b) sensor response to ac voltage of $200 \mathrm{~V}_{\text {rms }}$ at room temperature; (c) sensor instantaneous input-output characteristic; (d) sensor input-output rms characteristic.

\section{CONCLUSIONS}

In this paper, a preliminary investigation of the operation of a novel intesiometric dual FBG-based voltage sensor has been presented. The proposed sensor construction allows for the elimination of tunable filters or scanning lasers to perform signal detection, greatly simplifying the measurement system and potentially improving the speed of response. The preliminary voltage characterization has shown that the proposed sensor system is capable of measuring ac voltage. Future work will concentrate on sensor design improvements and characterization at higher temperatures.

\section{ACKNOWLEDGEMENTS}

Research presented in this paper was carried out within the European Metrology Research Programme (EMPR). The EMRP is jointly funded by the EMRP participating countries within EURAMET and the European Union.

\section{REFERENCES}

[1] Di Bisceglie, C. Galdi, A. Vaccaro, D. Villacci, "Cooperative sensor networks for voltage quality monitoring in smart grids," PowerTech, 1-6 (2009)

[2] J. Luszcz, R. Smolenski, "Voltage harmonic distortion measurement issue in smart-grid distribution system," APEMC, 841-844 (2012)

[3] Piezo Technologies, http://www.piezotechnologies.com (10 March 2015)

[4] G. Fusiek, P. Orr, P. Niewczas, 'Temperature-Independent High-Speed Distributed Voltage Measurement using Intensiometric FBG Interrogation", Proc. I2MTC, in press, (2015)

[5] P. Niewczas, L. Dziuda, G. Fusiek, J. R. McDonald, "Temperature Compensation for a Piezoelectric FibreOptic Voltage Sensor," Proc. IMTC, 1994-1998 (2006)

[6] G. Fusiek, P. Niewczas, J. R. McDonald, "Improved Method of Hysteresis Compensation for a Piezoelectric Fiber-Optic Voltage Sensor,” OE 46(3), 034401-1-8 (2007) 